\title{
A Comparative Study on Lithium Abundances in Solar-Type Stars With and Without Planets
}

\author{
Y. Q. Chen and G. Zhao \\ National Astronomical Observatories, Chinese Academy of Sciences, A20, Datun Road, \\ Chaoyang District, Beijing 100012, China; gzhao@bao.ac.cn
}

\begin{abstract}
We have investigated the abundance anomalies of lithium for stars with planets in the temperature range of 5600-5900 K reported by Israelian and coworkers, as compared to 20 normal stars at the same temperature and metallicity ranges. Our result indicates a higher probability of lithium depletion for stars with planets in the main-sequence stage. It seems that stellar photospheric abundances of lithium in stars with planets may be somewhat affected by the presence of planets. Two possible mechanisms are considered to account for the lower Li abundances of stars with planets. One is related to the rotation-induced mixing due to the conservation of angular momentum by the protoplanetary disk, and the other is a shear instability triggered by planet migration. These results provide new information on stellar evolution and the lithium evolution of the Galaxy.
\end{abstract}

Subject headings: planetary systems - stars: late-type - stars: abundances Galaxy: evolution

\section{Introduction}

Since the first announcement of a planet orbiting a Sun-like star, 51 Peg, by Mayor \& Queloz (1995), around 140 stars with planets (SWPs) have been reported. The main properties of SWPS have been investigated by many works. Among these, spectroscopic observations of most SWPs based on high-resolution and high signal-to-noise ratio spectra have been carried out. All of these works indicate a high mean metallicity of SWPs relative to normal field stars. Are there any other chemical signatures for SWPs? There is no conclusive result on this issue, and only a few works have reported abundance anomalies in some elements. For example, Gonzalez \& Laws (2000) suggested a high [C/Fe] ratio for SWPs as compared with the normal stars from Gustafsson et al. (1999). However, they later 
reported that there is no difference in abundance for $\mathrm{C}, \mathrm{Na}, \mathrm{Mg}$, and $\mathrm{Al}$ elements between SWPs and normal stars (Gonzalez et al. 2001). Present knowledge on the abundances of 25 elements (except for $\mathrm{Li}$ and $\mathrm{Be}$ ) available in the literature shows no special feature for SWPs except for the high overall metallicity.

For lithium abundance, Israelian et al. (2001) claimed that ${ }^{6} \mathrm{~L} i$ was detected in the atmosphere of HD 82943, while Reddy et al. (2002) did not confirm this detection. Mandell \& Ge (2004) reported no ${ }^{6} \mathrm{Li}$ presence in several lithium-poor stars. For ${ }^{7} \mathrm{Li}$, King et al. (1997) first found several SWPs with low Li abundances, and they related them with the presence of a planetary companion. Gonzalez \& Laws (2000) concluded that smaller Li abundances are found for SWPs after correcting for trends with temperature, metallicity, and age. However, Ryan (2000) suggested that there is no significant difference in lithium abundance for SWPs, and later work by Gonzalez et al. (2001) agreed with Ryan's conclusion. With a large sample, Israelian et al. (2004) suggested again that in the temperature range 5600-5850 K, SWPs show additional depletion of Li compared to normal stars. They also showed that for higher temperatures, the Li abundances were similar for SWPs and for normal stars. Recently, measurements of Be abundances by Santos et al. (2004) indicated further evidence on the composition difference between stars with and without planets.

Lithium is a special element because it is easily destroyed in stars during even pre-main sequence and main sequence evolution, which is not the case for other elements. Therefore, $\mathrm{Li}$ abundance reflects the mixing history of the star. For SWPs, Li is further related with the accretion of material and the angular-momentum evolution of the system, and thus Li abundances have been used to distinguish the current hypotheses on planet formation mechanisms. In this respect, it is important to verify whether Li abundance anomalies for SWPs at the temperature range of 5600-5850 K reported by Israelian et al. (2004) are real, which will provide new information on the planet formation process and its influence on host stars.

In this work we conduct a parallel abundance analysis of lithium abundances for 16 SWPs and 20 comparison stars in the same metallicity of $[\mathrm{Fe} / \mathrm{H}] \sim 0.1$ dex at the temperature range of $5600-5900 \mathrm{~K}$ in order to investigate whether any anomalies persist. Furthermore, this study is quite interesting for our understanding of the lithium behavior of solarmetallicity stars, which show a large scatter at $5600<T_{\text {eff }}<5900 \mathrm{~K}$ and which the standard model of stellar evolution cannot explain. With a consistent analysis procedure, this work aims to investigate the effect of planet presence on stellar evolution. This will provide new information on the chemical evolution of lithium in the Galaxy. 


\section{Observations and Abundance Analysis}

SWPs are selected from a catalog ${ }^{1}$ in the temperature range of 5600-5900 K, and their spectra are available from our observations with the $2.16 \mathrm{~m}$ telescope of the National Astronomical Observatories (Xinglong, China) with a resolving power of 37000 , or from Prugniel \& Soubiran (2001), who observed with the $1.93 \mathrm{~m}$ telescope of the Observatoire de Haute Provence with R 42000. Prugniel \& Soubiran (2001) present a database of highresolution spectra of 709 stars covering a large range of atmospheric parameters, which enables us to select a comparison sample of stars spanning the same ranges in temperature, gravity and metallicity as the above sample of planet stars but not having been reported to harbor planets. That is, both samples cover the atmospheric parameters of $5600<T_{\text {eff }}<$ $5900 \mathrm{~K}, \log g>3.8$, and $[\mathrm{Fe} / \mathrm{H}]>-0.3$. In the selection, strömgren uvby indices from Olsen $(1983,1993)$ are adopted and the metallicity is estimated based on the calibrations by Schuster \& Nissen (1989). Along with the initial metallicity, temperature and gravity are derived in the same way as described below. All spectra have signal-to-noise ratios above 150 pixel $^{-1}$ at the Li I $\lambda 6707$ line.

The spectra were reduced using standard MIDAS (for Xinglong data) and IRAF (for ELODIE data) routines for order definition, background correction, flat-fielding, extraction of echelle orders, wavelength calibration, and continuum fitting. The Fe II lines suitable for measurement were carefully selected, and the equivalent widths (EWs) were obtained by the

fitting of a Gaussian function. Atomic data for Fe II lines were the same as those in Chen et al. (2002). The wavelengths and oscillator strengths of the Li I $\lambda 6707$ line are taken from Smith et al. (1998).

\footnotetext{
${ }^{1}$ http://www.obspm.fr
} 
Table 1: Stellar Parameters for SWPs and the Comparison Stars

\begin{tabular}{|c|c|c|c|c|c|c|c|c|c|c|c|}
\hline $\begin{array}{l}\text { Star } \\
\text { HD }\end{array}$ & $\begin{array}{r}T_{\text {eff }} \\
\mathrm{K}\end{array}$ & $\log g$ & $\begin{array}{c}\xi_{t} \\
\mathrm{~km} \mathrm{~s}^{-1} \\
\end{array}$ & {$[\mathrm{Fe} / \mathrm{H}]$} & $\mathrm{Li}$ & $\begin{array}{l}\text { Age } \\
\text { Gyr }\end{array}$ & $\begin{array}{r}\mathrm{EW}(1) \\
\mathrm{mA}\end{array}$ & $\begin{array}{r}\sigma_{W} \\
\mathrm{~mA} \\
\end{array}$ & $\begin{array}{r}\mathrm{EW}(2) \\
\mathrm{mA} \\
\end{array}$ & $\begin{array}{r}\mathrm{EW}(3) \\
\mathrm{mA} \\
\end{array}$ & $\begin{array}{r}\mathrm{EW}(4) \\
\mathrm{mA} \\
\end{array}$ \\
\hline \multicolumn{12}{|c|}{ Stars with planets } \\
\hline Sun & 5780 & 4.44 & 1.15 & -0.03 & $<1.27$ & 4.6 & .. & 1.2 & $\ldots$ & $\ldots$ & $\ldots$ \\
\hline 12661 & 5682 & 4.26 & 1.50 & 0.30 & 1.74 & 9.6 & 16.6 & 0.8 & $\ldots$ & $\ldots$ & $\ldots$ \\
\hline 16141 & 5752 & 4.13 & 1.40 & 0.08 & $<1.03$ & 7.6 & $\ldots$ & 1.9 & $<2.0$ & $\ldots$ & $\ldots$ \\
\hline 23596 & 5906 & 4.09 & 1.60 & 0.31 & 2.78 & 5.9 & 86.7 & 1.6 & $\ldots$ & $\ldots$ & $\ldots$ \\
\hline 33636 & 5817 & 4.41 & 1.30 & -0.03 & 2.29 & 5.8 & 40.3 & 1.5 & 49.0 & $\ldots$ & $\ldots$ \\
\hline 72659 & 5846 & 4.16 & 1.15 & 0.04 & 2.25 & 6.3 & 35.4 & 0.6 & $\ldots$ & $\ldots$ & $\ldots$ \\
\hline 82943 & 5858 & 4.32 & 1.40 & 0.20 & 2.37 & 6.0 & 44.7 & 2.2 & 44.1 & $\ldots$ & $\ldots$ \\
\hline 92788 & 5683 & 4.36 & 1.40 & 0.18 & $<0.97$ & 6.6 & $\ldots$ & 1.7 & $\ldots$ & $\ldots$ & $\ldots$ \\
\hline 95128 & 5755 & 4.23 & 1.30 & -0.00 & 1.68 & 9.3 & 10.5 & 2.0 & 12.5 & $\ldots$ & 18.0 \\
\hline 106252 & 5769 & 4.32 & 1.00 & 0.01 & 1.72 & 9.6 & 13.5 & 2.1 & $\ldots$ & $\ldots$ & $\ldots$ \\
\hline 134987 & 5735 & 4.27 & 1.15 & 0.24 & $<1.02$ & 8.3 & $\ldots$ & 1.2 & $<2.0$ & $\ldots$ & $\ldots$ \\
\hline 143761 & 5701 & 4.26 & 1.20 & -0.21 & 1.37 & 10.8 & 6.2 & 1.1 & 5.9 & $\ldots$ & 6.0 \\
\hline 150706 & 5764 & 4.43 & 1.20 & 0.01 & 2.40 & 7.5 & 53.3 & 1.4 & 55.4 & $\ldots$ & $\ldots$ \\
\hline 187123 & 5717 & 4.26 & 1.15 & 0.11 & $<1.00$ & 8.8 & $\ldots$ & 1.8 & $\ldots$ & $\ldots$ & $\ldots$ \\
\hline 195019 & 5729 & 4.07 & 1.15 & 0.03 & 1.55 & 7.3 & 9.9 & 1.8 & 6.9 & $\ldots$ & $\ldots$ \\
\hline 217014 & 5654 & 4.27 & 1.15 & 0.20 & $<0.89$ & 10.4 & $\ldots$ & 0.8 & 4.4 & $\ldots$ & $\ldots$ \\
\hline \multicolumn{12}{|c|}{ The comparison stars } \\
\hline 4307 & 5748 & 3.95 & 1.30 & -0.20 & 2.38 & 6.9 & 53.9 & 1.9 & 52.3 & $\ldots$ & $\ldots$ \\
\hline 4614 & 5818 & 4.31 & 1.20 & -0.23 & 2.08 & 10.3 & 21.7 & 1.0 & 26.3 & 24.0 & 21.0 \\
\hline 9562 & 5786 & 3.99 & 1.20 & 0.18 & 2.51 & 4.6 & 61.0 & 1.7 & 61.2 & $\ldots$ & $\ldots$ \\
\hline 15335 & 5797 & 3.93 & 1.40 & -0.15 & 2.55 & 7.0 & 65.7 & 0.7 & $\ldots$ & 57.9 & 52.0 \\
\hline 34411 & 5800 & 4.24 & 1.20 & 0.07 & 2.07 & 7.2 & 24.3 & 1.1 & 25.7 & 27.4 & $\ldots$ \\
\hline 39587 & 5833 & 4.41 & 1.40 & -0.00 & 2.85 & 5.8 & 103.9 & 1.1 & 101.3 & 105.6 & 95.0 \\
\hline 52711 & 5778 & 4.31 & 1.30 & -0.10 & 1.84 & 9.2 & 17.2 & 1.5 & 17.5 & $\ldots$ & $\ldots$ \\
\hline 70110 & 5880 & 3.93 & 1.40 & 0.10 & 2.49 & 3.9 & 53.2 & 1.9 & 52.0 & 55.8 & $\ldots$ \\
\hline 76151 & 5702 & 4.39 & 1.20 & 0.05 & 1.80 & 6.0 & 18.2 & 1.6 & 18.7 & $\ldots$ & $\ldots$ \\
\hline 79028 & 5818 & 4.05 & 1.50 & 0.03 & 2.65 & 7.2 & 76.1 & 1.4 & 74.1 & 76.5 & 11.0 \\
\hline 84737 & 5822 & 4.11 & 1.30 & 0.17 & 2.32 & 5.7 & 42.9 & 1.5 & 41.8 & $\ldots$ & $\ldots$ \\
\hline 88986 & 5750 & 4.09 & 1.30 & -0.02 & 1.97 & 7.2 & 23.6 & 1.5 & 26.2 & $\ldots$ & $\ldots$ \\
\hline 109358 & 5742 & 4.28 & 1.20 & -0.26 & 1.59 & 12.0 & 10.7 & 0.8 & 9.3 & $\ldots$ & 9.0 \\
\hline 114710 & 5920 & 4.39 & 1.30 & 0.13 & 2.56 & 2.3 & 63.3 & 1.0 & 57.8 & $\ldots$ & $\ldots$ \\
\hline 115383 & 5918 & 4.19 & 1.30 & 0.19 & 2.78 & 5.2 & 79.2 & 1.3 & 83.1 & $\ldots$ & $\ldots$ \\
\hline 141004 & 5806 & 4.17 & 1.40 & -0.02 & 1.79 & 8.2 & 14.7 & 1.5 & 16.0 & $\ldots$ & 20.0 \\
\hline 182572 & 5648 & 4.12 & 1.40 & 0.15 & $<0.90$ & 8.8 & & 2.4 & $<1.0$ & $\ldots$ & $\ldots$ \\
\hline 190406 & 5797 & 4.38 & 1.40 & 0.04 & 2.26 & 5.6 & 39.4 & 1.8 & 37.6 & $\ldots$ & $\ldots$ \\
\hline 193664 & 5795 & 4.39 & 1.20 & -0.11 & 2.27 & 6.1 & 39.9 & 1.2 & 34.0 & $\ldots$ & $\ldots$ \\
\hline 196755 & 5675 & 3.66 & 1.40 & -0.04 & 1.28 & 3.0 & 6.6 & 1.3 & 10.8 & $\ldots$ & $\ldots$ \\
\hline
\end{tabular}


Effective temperatures were determined from the Strömgren $(b-y)$ color index (Olsen 1983, 1993) using the calibration of Alonso et al. (1996). The gravities were determined via Hipparcos parallaxes (Perryman et al. 1997), and the microturbulences were obtained by forcing Fe I lines with different strengths to give the same abundances. Here the oscillator strengths of the Fe I lines are taken from Chen et al. (2000). The new metallicity derived from spectroscopic analysis was updated, and the procedure of atmospheric parameter determination was iterated for consistency, even though there is no systematic difference between the initial metallicity and the new value, with a deviation of $0.01 \pm 0.15$ dex. The internal errors of the atmospheric parameters are around $70 \mathrm{~K}$ in temperature, 0.1 dex in $\log g, 0.1$ dex in $[\mathrm{Fe} / \mathrm{H}]$, and $0.3 \mathrm{~km} \mathrm{~s}^{-1}$ in microturbulence. But the absolute uncertainties of the stellar parameters may be slightly larger.

We have found that the photometry-based temperatures in the work are $130 \mathrm{~K}$ lower than the spectroscopically-derived values in Santos et al. (2004) for SWPs in common. However, our temperatures are $83 \mathrm{~K}$ higher than those in Takeda \& Kawanomoto (2005), who derive temperatures by spectroscopic method for 29 stars in common. It seems that spectroscopically-derived temperatures have significant uncertainties, and the values depend on stellar model atmosphere, the selection of lines, the adopted atomic data, and the way the temperatures are determined. In addition, as already noted by Santos et al. (2004), surface gravities determined from the balance of Fe I and Fe II abundances are systematically higher than those derived from parallaxes (adopted in our work) by the order of $0.15-0.20$ dex. However, iron abundances in this work and Santos et al. (2004) are similar since we adopted iron abundances from Fe II lines and they forced Fe I and Fe II lines to give the same iron abundances when they derived gravities. For the comparison stars, there are 15 stars in common with Edvardsson et al. (1993), and our temperatures are $87 \mathrm{~K}$ lower than their values. This is due to different temperature calibrations, and our values, based on the infrared flux method temperatures by Alonso et al. (1996), may be more reliable. Actually, the absolute temperature and gravity are not very important, since we are carrying out a differential analysis, and it is crucial that the stellar parameters all be in the same scale between the target and the comparison sample.

The model atmospheres were interpolated from a grid of plane-parallel, LTE models provided by Kurucz (1995) in which convective overshoot is switched off. The ABONTEST8 program developed by P. Magain in the Liége group was used to carry out the calculations of theoretical line EWs, and abundances were derived by matching the theoretical EWs to the observed values. Abundance errors, estimated from the uncertainties of atmospheric parameters and EWs, are 0.07 dex for iron abundances, and less than 0.1 dex for Li abundances. Here the contribution of the ${ }^{6} \mathrm{Li}$ isotope to the $\mathrm{Li}$ I $\lambda 6707$ line was assumed to be negligible. Finally, non-LTE corrections were applied to the derived Li abundances based on the work 


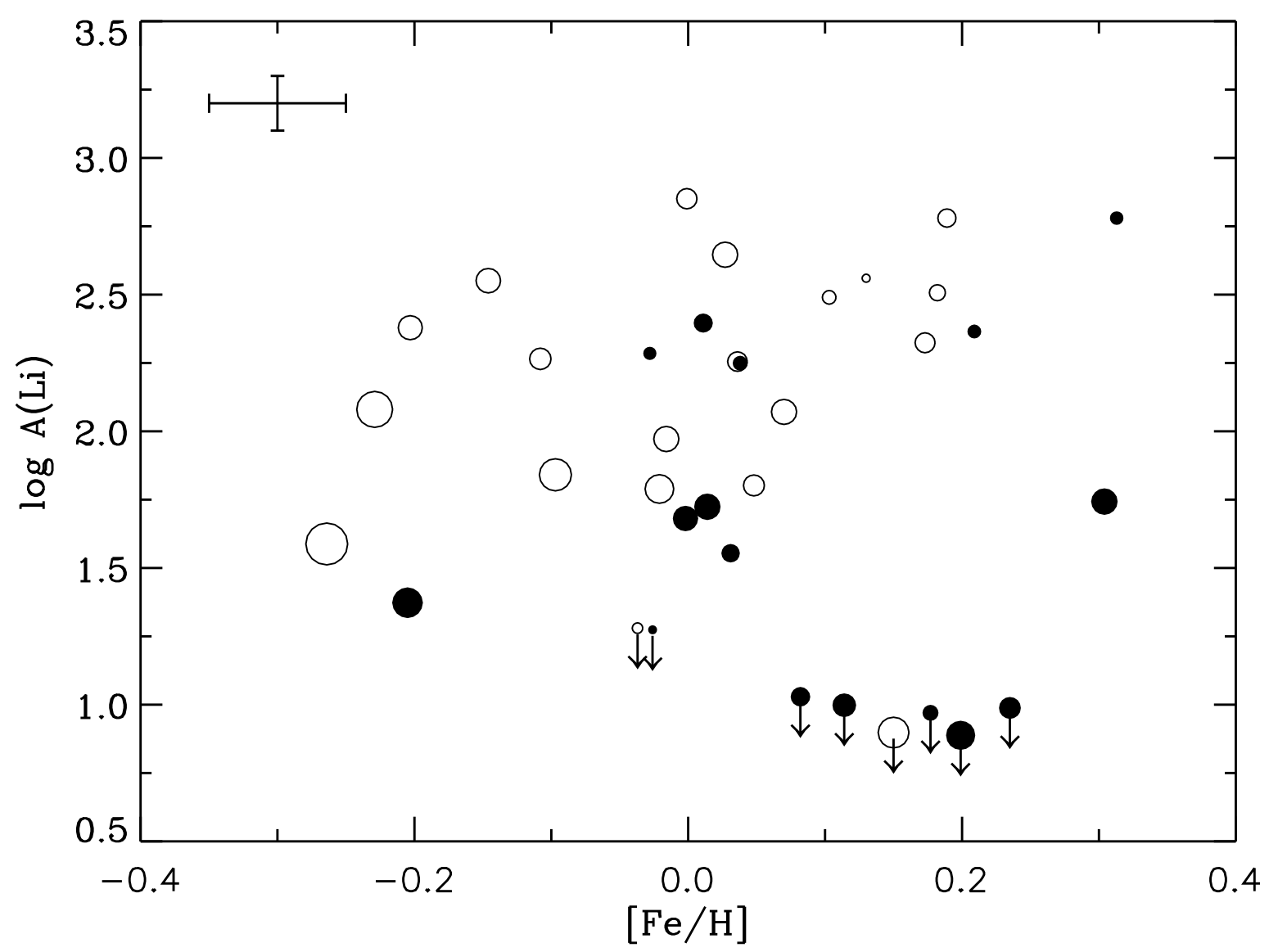

Fig. 1.- Li abundances vs. $[\mathrm{Fe} / \mathrm{H}]$ for stars with (filled circles) and without (open circles) planets. Upper limits of Li abundances are indicated by downward-directed arrows, and the size of the symbol corresponds to stellar age. 


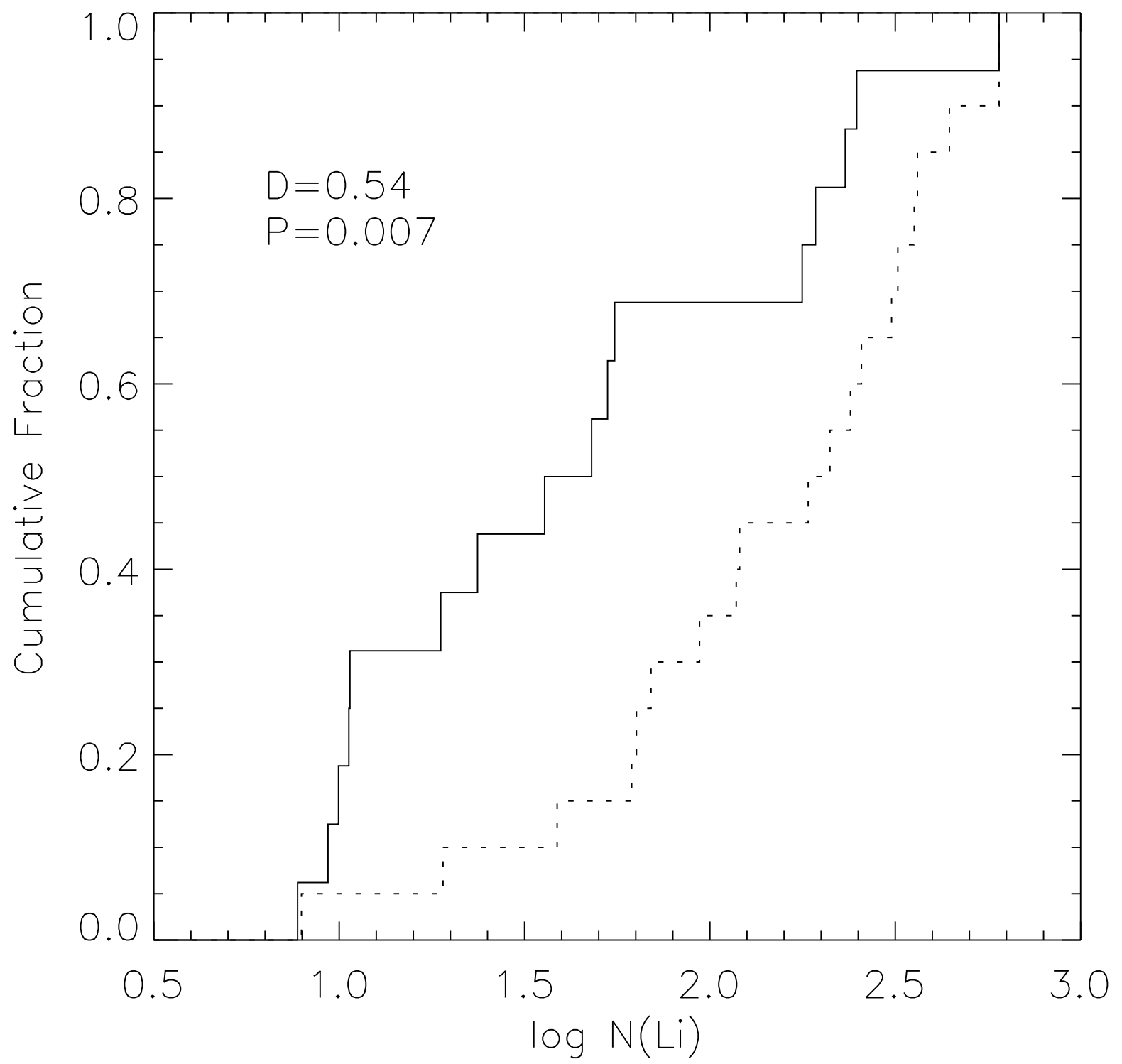

Fig. 2.- Cumulative distribution functions based on the Kolmogorov-Smirnov test for stars with (solid line) and without (dash line) planets. Shown are different groups between the two samples, with the maximum value of $\mathrm{D}=0.54$ and the probability for the two samples being the same group of 0.007 . 


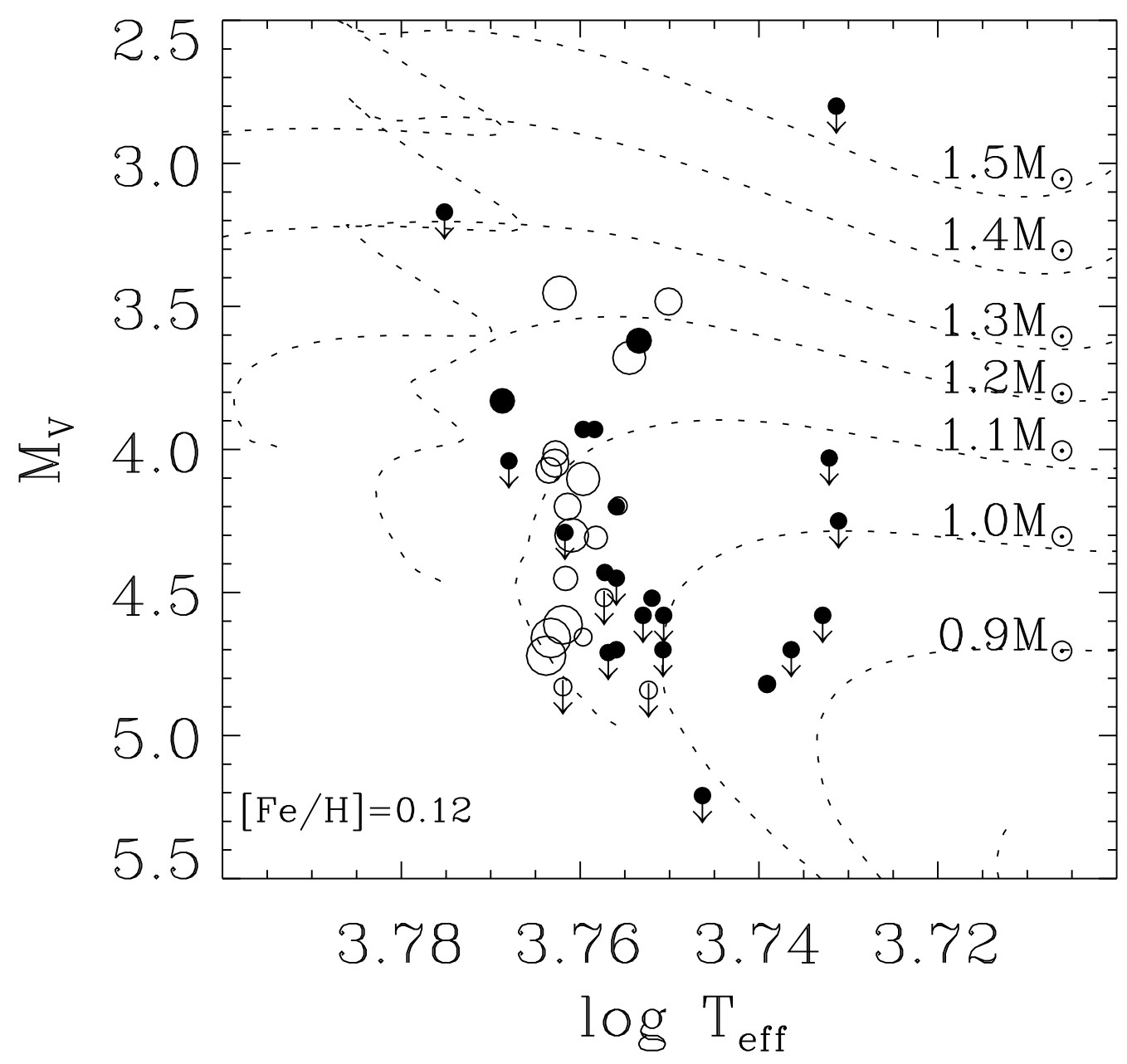

Fig. 3.- Stellar positions of SWPs in Israelian et al. (2004) (filled circles) with new determined temperatures as compared with Chen et al. (2001; open circles) for stars with $5600<T_{\text {eff }}<5850 \mathrm{~K}$ and $[\mathrm{Fe} / \mathrm{H}]>-0.3$. Downward-directed arrows correspond to stars with upper limits for Li abundances, and diameters of circles correspond to stellar Li abundances taken directly from Israelian et al. (2004) and Chen et al. (2001). 


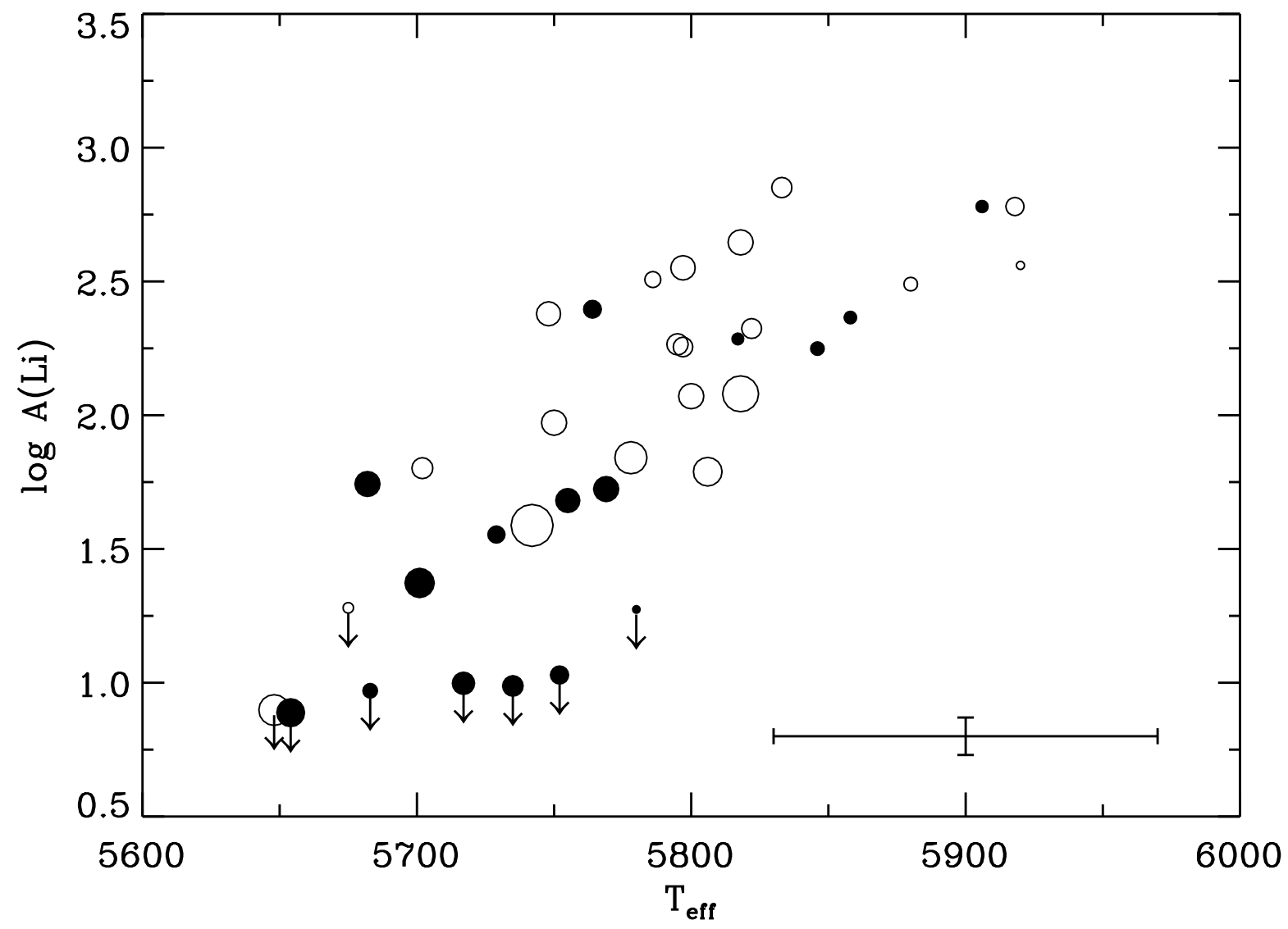

Fig. 4. - Li abundance vs. $T_{\text {eff }}$. The symbols are the same as in Fig. 1. 


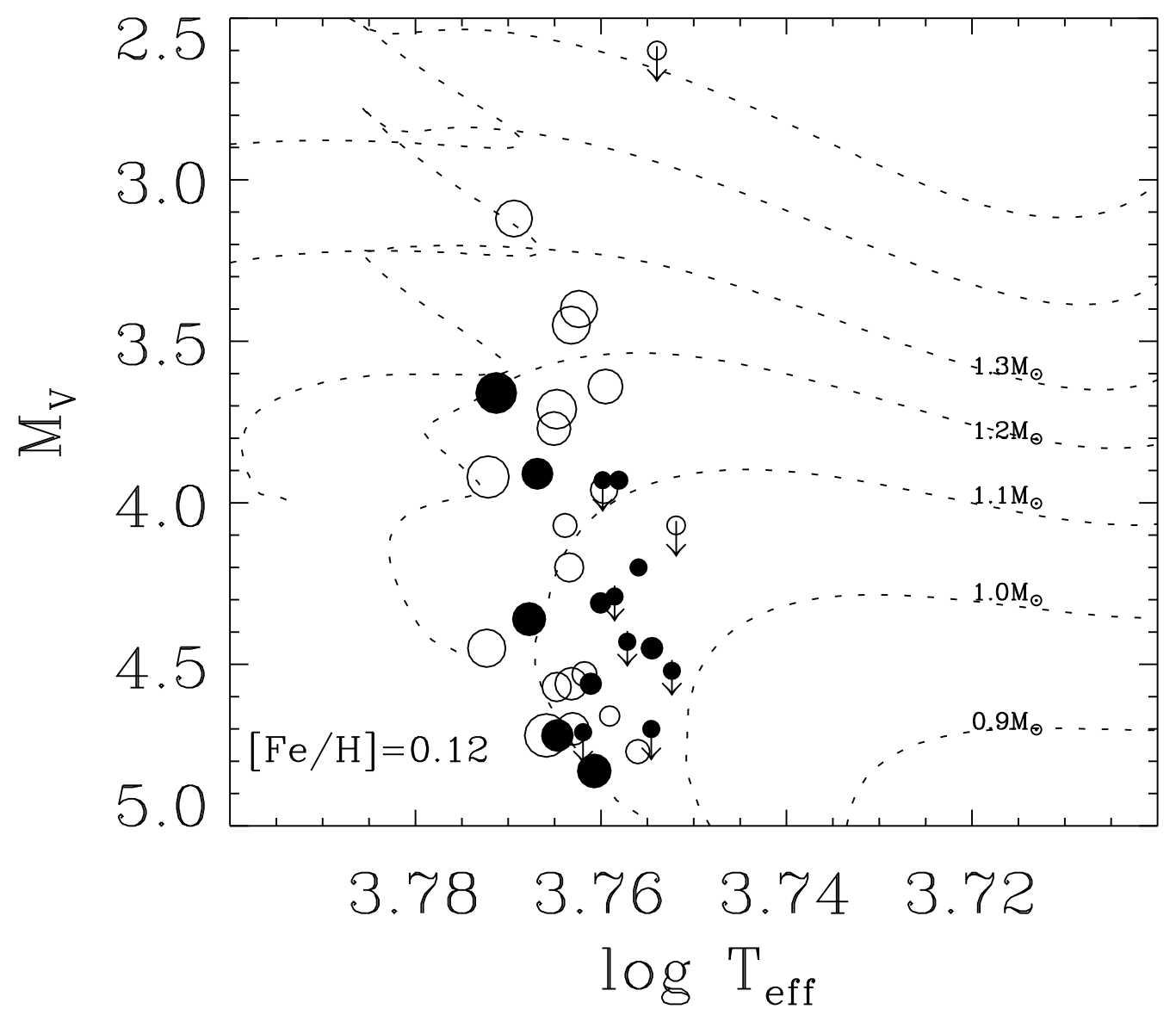

Fig. 5.- Stellar positions in the HR diagram as compared with the evolution tracks from VandenBerg et al. (2000). The symbols are the same as in Fig. 1, but diameters of circles correspond to Li abundances. 
by Carlsson et al. (1994), who studied non-LTE formation of the Li I $\lambda 6708$ line as a function of effective temperature, gravity, metallicity, and $\mathrm{Li}$ abundance. Stellar parameters and Li and Fe abundances for SWPs and the comparison stars, as well as the EWs of the lines and their errors are shown in Table 1.

\section{Results and Discussions}

\subsection{Li Abundances versus $[\mathrm{Fe} / \mathrm{H}]$}

$\mathrm{Li}$ abundances as a function of metallicity for all stars in the present survey are shown in Fig. 1, in which stars with downward-directed arrows have an undetected lithium line, and the symbol size corresponds to stellar age. The upper limits of the Li abundances are estimated by assuming EWs of $3.0 \mathrm{~m} \AA$, which is the estimated error in EW from the comparison used in Chen et al. (2000). Based on Table 1, the scatter of the EW comparison between this work and Takeda \& Kawanomoto (2005), Chen et al. (2001), and Lambert et al. (1991) is around $4.0 \mathrm{~m} \AA$, which indicates an error of $4.0 / \sqrt{2} \sim 3.0 \mathrm{~m} \AA$ assuming the same uncertainties for the EWs from the other works. Note that the statistical uncertainties based on the formula by Cayrel (1988), presented in Table 1, are smaller. Since experimental errors by different authors during spectrum normalization and EW measurements are not considered in Cayrel's (1988) formula, we believe that an upper limit to the EWs of $3.0 \mathrm{~m} \AA$ for stars with an undetected Li line may be more reasonable.

An interesting result from Fig. 1 is that there is a lack of normal stars with $[\mathrm{Fe} / \mathrm{H}]>$ +0.2 . This suggestion is supported by large samples of stars in the literature. There are $22 \%$ normal stars with $[\mathrm{Fe} / \mathrm{H}]>+0.2$ in Valenti \& Fischer (2005)'s sample at the temperature range of 5600-5900 K, but note that their temperatures, derived from the fitting of synthetic spectra with observed spectra, are $107 \mathrm{~K}$ higher than our values for stars in common, and the sample is too small for statistics. With photometry-based temperatures and a larger sample of stars from Nordström et al. (2004), this fraction is reduced to $2 \%$, indicating a low probability of super-metal-rich stars in the solar neighbourhood. This is probably due to the fact that most metal-rich stars tend to have formed planetary systems, and thus it is difficult to obtain a comparison sample that consists of super-metal-rich stars not harboring a planet. This is consistent with the current knowledge that SWPs have higher mean metallicities than normal stars.

The second feature from Fig. 1 is that six of the 16 SWPs versus two of the 20 normal stars have undetected lithium lines. All of the six SWPs with undetected Li lines are mainsequence stars while the two normal stars with depleted Li abundances are subgiants, as 
shown below (Fig. 5). It seems that main-sequence SWPs in the temperature range of 5600$5900 \mathrm{~K}$ destroy their Li much more easily than do normal stars. A Kolmogorov-Smirnov test is applied to stars with and without planets, and the cumulative distribution functions are shown in Fig. 2. It shows that the maximum value of the absolute difference between the two distribution functions is 0.54 , and the probability for the two samples being the same group is 0.007. This is also evident from Israelian et al. (2004), as shown in Fig. 3, in which eight of 16 main-sequence SWPs at $5600<T_{\text {eff }}<5850 \mathrm{~K}$ only have upper limits for Li abundances, while three of the 14 normal main-sequence stars from Chen et al. (2001) are depleted in Li. Note that the two comparison samples, from this work and Chen et al. (2001), are independently selected with six common stars. Thus, this property could be intrinsic, but further investigations with large samples of both SWPs and normal stars are needed.

If this suggestion that main-sequence SWPs have a higher probability of depleting their lithium abundances is true, it will provide new information on stellar evolution for SWPs. We suspect that the stellar evolution history between SWPs and normal stars could be somewhat different due to the presence of a planet, which appears to affect only Li abundances, with no other elements showing any difference. As suggested by King et al. (1997), the presence of planets or associated circumstellar disks may affect a parent star's initial angular momentum and/or subsequent evolution. Specifically, the conservation of angular momentum in the protoplanetary disk may induce increased mixing by causing rotational breaking in the host stars during the pre-main-sequence evolution. This effect, once it happens, will destroy the Li completely. Considering this suggestion as one possibility, Israelian et al. (2004) proposed a second hypothesis: that migration triggers tidal forces and creates a shear instability that leads to a strong depletion of $\mathrm{Li}$ abundance. Both mechanisms occur during the pre-mainsequence evolution, and thus they can be used to explain our result. Further theoretical and observational studies are very desirable to investigate whether the two mechanisms are reasonable.

\subsection{Li Aabundances versus $T_{\text {eff }}$}

Fig. 4 shows Li abundances versus temperatures for all stars. It seems that $\mathrm{Li}$ abundance generally increases with increasing $T_{\text {eff }}$, and there is no significant gap between SWPs and normal stars at a given temperature. Fig. 5 shows the stellar positions of the two samples of stars in the HR diagram as compared with the evolutionary tracks of VandenBerg et al. (2000) with $[\mathrm{Fe} / \mathrm{H}] \sim+0.12$ dex. Stellar ages are also estimated from this comparison, and they are presented in Table 1. The error of the ages due to the uncertainties of $T_{\text {eff }}, M_{V}$, and $[\mathrm{Fe} / \mathrm{H}]$ is about $15 \%$. It is clear that our two samples of stars are generally located in 
the same regions of the HR diagram, and during the selection all these stars are classified as main-sequence stars with similar parameters. However, the two stars from the comparison sample showing depleted Li abundances, as indicated by downward-directed arrows in Fig. 5, are actually subgiants. Other possible subgiant stars are not evolved far away from the main sequence, and there is no difference in Li abundance between SWPs and normal stars.

This observation that subgiants tend to have depleted Li abundances reminds us to investigate the stellar positions of SWPs at $5600<T_{\text {eff }}<5850 \mathrm{~K}$ in Israelian et al. (2004). Here we redetermined the temperatures for SWPs with $5600<T_{\text {eff }}<5850 \mathrm{~K}$ from Israelian et al. (2004) in the same way as Chen et al. (2001). In Fig. 3, the stellar positions of these SWPs with new temperatures are plotted against normal stars from Chen et al. (2001) in this temperature range with $[\mathrm{Fe} / \mathrm{H}]>-0.3$. It is clear that a few SWPs in Israelian et al.'s (2004) sample based on the updated temperatures are actually evolved subgiants, and downward-directed arrows in Fig. 3 indicate stars with undetected Li I $\lambda 6708$ lines. The fact that evolved subgiants tend to have depleted Li abundances is consistent with the knowledge of stellar evolution theory.

It has been suggested in Ryan (2000) that the Li abundances of subgiants can be somewhat depleted due to the dilution proces without complete destruction, as opposed to mainsequence stars. In this scenario, once a star evolves onto the subgiant branch, it dilutes surface Li when convection brings Li-poor material from deep layers to the surface. This dilution is not obvious for slightly evolved subgiants in the present study, and a few subgiants with $1.2 M_{\odot}$ show higher Li abundances than main-sequence stars. Moreover, there is no difference in Li abundance between planet hosts and normal stars on the subgiant branch. Actually, the dilution process mentioned above is difficult to detect, and its effect is masked by the large scatter of $\mathrm{Li}$ abundances for even slightly evolved subgiants. It is impossible to investigate how the presence of a planet will affect lithium evolution for evolved subgiants in the present work, since their Li abundances have already been depleted due to their low temperatures.

As shown in Fig. 4, there is a substantial scatter of Li abundances at $T_{\text {eff }} \sim 5800 \mathrm{~K}$. Considering the uncertainty in temperature, it is difficult to investigate whether the scatter is real. There seems to be a tendency that stars in the upper envelope of the Li versus $T_{\text {eff }}$ diagram seem to be slightly younger than stars with the lowest Li abundances in the $T_{\text {eff }}<5800 \mathrm{~K}$ range. However, since there is a lack of young stars in the low-temperature range and of old stars at the high-temperature edge in our sample, we refrain from drawing any firm conclusions. A larger sample of stars with different ages at a given temperature is needed to clarify this issue. 


\subsection{Li Anomalies of Stars With Planets and the Planet Formation}

The excess of Li depletion in SWPs in the temperature range of $5600<T_{\text {eff }}<5850 \mathrm{~K}$ suggested by Israelian et al. (2004) is based on the fact that Li abundances for SWPs are lower than for comparison stars. A closer comparison shows that the average of Li abundances for 24 SWPs at this temperature range in Israelian et al. (2004) is $<0.99 \pm 0.45$, while this value is $2.08 \pm 0.49$ for the comparison stars from Chen et al. (2001). In our work the average $\mathrm{Li}$ abundance is $<1.65 \pm 0.57$ for SWPs versus $2.15 \pm 0.49$ for the comparison stars. As presented above, this anomaly in $\mathrm{Li}$ abundance of SWPs might reflect the effect of planet presence on stellar evolution and cannot be used to provide constraints on planet formation. In addition, this effect is difficult to detect from stars in the subgiant branch or later stages if their Li abundances have already been depleted by other mechanisms. Moreover, the deviations of the average $\mathrm{Li}$ abundance between stars with and without planets are 0.27 dex for $T_{\text {eff }}>5800 \mathrm{~K}$ and 0.58 dex for $T_{\text {eff }}<5800 \mathrm{~K}$. It seems that Li anomalies concentrate in the range of $T_{\text {eff }}<5800 \mathrm{~K}$, and thus they do not relate to the Li-dip in the Hyades, which happens in the temperature range of $6300-6600 \mathrm{~K}$ as first found by Boesgaard \& Tripicco (1986).

In connection with planet formation mechanism, our result does not support the accretion scenario of planet formation. Theoretically, Montallbán \& Rebolo (2002) predicted quantitative estimates of the main-sequence evolution of stellar surface lithium after planet ingestion. They found that the preservation of ${ }^{7} \mathrm{~L} i$ occurs in a large mass range of $0.9-1.3$ $M_{\odot}$ at solar metallicity. For SWPs with a typically super-solar metallicity, the mass range is slightly reduced. According to their theory, if a $1 M_{\mathrm{J}}$ planet were dissolved in the convective envelope of an SWP at a sufficiently old age for extra-mixing to be inefficient, it would produce an enhancement of 0.3 dex in Li abundance, which is unfortunately within the scatter of $\mathrm{Li}$ abundance at the temperature range we investigated. But Li abundances for stars with $T_{\text {eff }}>5900 \mathrm{~K}$ in Israelian et al. (2004) do not show such a large scatter, and the similar abundance between SWPs and the comparison stars argues against the accretion scenario. Furthermore, most SWPs known so far have giant planets with masses larger than $5 M_{\mathrm{J}}$, and if $10 M_{\mathrm{J}}$ were engulfed the enhancement would reach 0.9 dex, which is easily detected. Therefore, we favor the scenario that the surface composition of SWPs is not polluted by the accretion of planetary material, and the high mean metallicity of SWPs is primordial.

\section{Conclusions}

We have made a parallel abundance analysis for 16 SWPs and 20 normal stars at the metallicity of $-0.2<[\mathrm{Fe} / \mathrm{H}]<+0.4$ and the temperature range of $5600<T_{\text {eff }}<5900 \mathrm{~K}$. 
Our results show that there is a higher probability for SWPs to have depleted Li abundances than for comparison stars, which confirms the possible Li abundance anomalies for SWPs at this temperature range reported by Israelian et al. (2004) after excluding evolved subgiants. We proposed that the presence of planets around host stars may affect stellar evolution by inducing additional mixing or shear instability, which leads to a high probability of destroying surface lithium while other elements are not affected. This effect can only be detected for main-sequence SWPs since other depletion mechanisms would not have started to act on main-sequence stars. In view of this, the effect of planet presence should be considered when the large scatters of the Galactic lithium in field disk stars at the temperature range of $5600<T_{\text {eff }}<5900 \mathrm{~K}$ are investigated.

\section{Acknowledgments}

This work is supported by the National Natural Science Foundation of China under grants 10433010 and 10203002 .

\section{REFERENCES}

Alonso, A., Arribas, S. \& Martínez-Roger, C. 1996, A\&AS, 117, 227

Boesgaard, A.M., \& Tripicco, M.J. 1986, ApJ, 302, L49

Carlsson, M., Rutten, R.J., Bruls, J.H.M.J., \& Shchukina, N.G. 1994, A\&A, 288, 860

Cayrel, R. 1988, IAU symp., 132, 345

Chen, Y.Q., Nissen, P.E., Zhao, G. et al., 2000, A\&AS, 141, 491

Chen, Y.Q., Nissen, P.E., Benoni, T., \& Zhao, G. 2001, A\&A, 371, 943

Chen, Y.Q., Nissen, P.E., Zhao, G., \& Asplund M. 2002, A\&A, 390, 925

D’Antona, F., \& Mazzitelli, 1994, ApJS, 90, 467

Edvardsson, B., Anderson, J., Gustafsson B., Lambert, D.L., Nissen, P.E., Tomkin, J., 1993, A\&A, 275, 101

Gonzalez, G., \& Laws, C. 2000, ApJ, 119, 390

Gonzalez, G., Lawsi, C., Tyagii, S., \& Reddy, B.E. 2001, AJ, 285, 403 
Gustafsson, B., Karlsson, T., Olsson, E., Edvardsson, B., \& Ryde, N. 1999, A\&A, 342, 426

Israelian, G., Santos, N., Mayor, M, \& Rebolo, R. 2001, Nature, 411, 163

Israelian, G., Santos, N., Mayor, M, \& Rebolo, R. 2004, A\&A, 414, 211

King, J.R., Deliysnnis, C.P., Hiltgen, D.D. et al., 1997, AJ, 113, 1871

Kurucz, R.L., 1995, CDROM, No. 23

Lambert, D.L., Heath, J.E., \& Edvardsson, B., 1991, MNRAS, 253, 610

Mandell, A.M., \& Ge, J. 2004, AJ, 127, 1147

Mayor, M., \& Queloz, D. 1995, Nature, 378, 357

Montalbán, J., \& Rebolo, R. A\&A, 386, 1043

Nordström, B., Mayor, M., Andersen, J., et al., 2004, A\&A, 418, 989

Olsen E.H., 1983, A\&AS 54, 55

Olsen E.H., 1993, A\&A 102, 89

Perryman, M.A.C. et al., 1997, The Hipparcos and Tycho Catalogs (ESA SP-1200, Noordwijk:ESA)

Prugniel, P., \& Soubiran, C. 2001, A\&A, 369, 1048

Reddy, B., Lambert, D.L., Laws, C., Gonzalez, G., \& Covery, K. 2002, ApJ, 572, 1012

Ryan, R.G. 2000, MNRAS, 316, L35

Santos, N.C., Israelian, G., García Lópex, G.J et al., 2004, A\&A, 427, 1085

Schuster, B.W., \& Nissen, P.E. 1989, A\&A, 221, 65

Smith, V.V., Lambert, D.L., \& Nissen, P.E. 1998, ApJ, 506, 405

Takeda, Y., \& Kawanomoto, S., 2005, PASJ, 57, 45

Valenti, J.A, \& Fischer, D.A., 2005, ApJS, 159, 141

VandenBerg, D.A., Swenson, F.J., Rogers, F.J., Iglesias, C.A., \& Alexander, D.R. 2000, ApJ, 532, 430 